\section{Natural Triterpene Glycosides for Antibody Recognition}

\author{
Elisa Peroni ${ }^{1,3}$, Feliciana Real Fernández ${ }^{1,2}$, Caterina Gheri ${ }^{1,2}$, \\ Francesca Nuti ${ }^{1,2}$, Anne-Claire Mitaine-Offer ${ }^{4}$, \\ Francesco Lolli ${ }^{1,5}$, Marie-Aleth Lacaille-Dubois ${ }^{4}$, \\ Anna-Maria Papini ${ }^{1,2,3}$ \\ ${ }^{1}$ Laboratory of Peptide and Protein Chemistry and Biology, University \\ of Florence, Sesto Fiorentino, Italy \\ 2 Department of Chemistry "Ugo Schiff" and NEUROFARBA Depart- \\ ment, Section of Pharmaceutical Sciences and Nutraceutics, Univer- \\ sity of Florence, Sesto Fiorentino, Italy \\ 3 Laboratory of Chemical Biology EA 4505 \& PeptLab@UCP, Université \\ de Cergy-Pontoise, Cergy-Pontoise, France \\ ${ }^{4}$ Laboratoire de Pharmacognosie, EA 4267 FDE, Université de Bour- \\ gogne Franche-Comté, Dijon, France \\ ${ }^{5}$ Department of Biomedical, Experimental and Clinical Sciences, Uni- \\ versity of Florence, Firenze, Italy
}

\section{Abstract \\ $\nabla$}

Multiple sclerosis is an autoimmune disease that affects the central nervous system. The key role of the glycosylation in disease pathogenesis has been previously studied and the synthetic Nglucosylated peptide CSF114(Glc) proved its efficiency in autoantibody recognition in the sera of multiple sclerosis patients. Herein, pure natural triterpene glycosides containing different glycosyl moieties were isolated and tested in multiple sclerosis patients' sera to better understand the role of glycosylation. They were selected taking into account the nature and complexity of their osidic part. Five triterpene glycosides were isolated from several plants with more than 95\% purity. The interaction between these glycosides and autoantibodies was evaluated in an enzyme-linked immunosorbent assay measuring IgG and IgM levels in multiple sclerosis patients and healthy blood donors, and the results were compared with those of CSF114(Glc). For each antigen, Mann Whitney and receiver operating characteristic-based analysis were performed to evaluate any statistical significance of the results. The five natural triterpene glycosides showed good capacity to recognize IgMs (sensitivity up to $38 \%$ ) with a high specificity (88.9\%) for multiple sclerosis patients.

Key words

multiple sclerosis · triterpene glycosides · autoimmune disease - biomarkers $\cdot$ autoantibody recognition

Supporting information available online at http://www.thieme-connect.de/products

Complex glycoconjugates, including glycolipids and glycoproteins, are commonly displayed on cell surfaces, where they play crucial roles as intermediaries in extracellular communication and signalling networks [1-3]. Therefore, studies on glycan modification patterns of glycoconjugates are a great challenge in medical diagnostic research, offering insight into cellular molecular mechanisms. Moreover, glycoconjugates can be proposed as molecular biomarkers for the disease activity $[4,5]$. In the field of autoimmunity, different approaches are used to investigate the structures and functions of complex naturally occurring glyco- conjugates leading to the identification of autoantigens associated with different autoimmune diseases. In particular, in the context of multiple sclerosis (MS), the development of serum antibodies as biomarkers to be used for the diagnosis, monitoring, and prognosis of such a heterogeneous disease is a challenge because of the ambiguous identification of antigens (Ags) implicated in the disease.

MS pathogenesis has not been yet elucidated but includes viral, immunological, and vascular hypotheses [6]. Most of the putative Ags belong to the myelin protein family (i.e., myelin basic protein, proteolipid lipoprotein, and myelin oligodendrocyte glycoprotein). Moreover, pathophysiological post-translational modifications of native Ags can trigger the immune system to generate autoantibodies escaping conventional diagnostic procedures [7]. Thus, autoantibodies against aberrant glycosylations have been hypothesized as diagnostic biomarkers in MS $[8,9]$. In particular, the N-glucosylated peptide CSF114(Glc) detecting disease-specific autoantibodies as biomarkers of MS has been developed as a probe for the detection of autoAbs present in the serum of MS patients $[10,11]$. This probe is able to identify and measure MS-related autoAbs whose levels correlated with a clinical assessment of MS activity and MRI profile of brain lesions [8]. The CSF114 (Glc) peptide represents an unconventional approach since its structure is completely unrelated to any primary myelin protein structure and is not linked to any particular pathogenetic hypothesis. The main characteristic of CSF114(Glc) is its conformational $\beta$-turn structure exposing the sugar moiety on an Asn residue, possibly the key element, for Ab recognition [12,13]. Different synthetic sugar moieties were assembled into the amino acid sequence of CSF114 and their reactivity studied with MS patients' sera, confirming the role of N-glucosylation in the autoAb recognition [14]. On the other hand, the nature of the native antigen bearing the sugar moiety has not been deeply investigated. With all these considerations in mind, structures containing naturally occurring glycosylations are attractive as possible antigenic probes mimicking native glycoconjugates. Triterpene glycosides are good candidates for this purpose due to their aglycon bearing various glycosidic moieties, from a single sugar to an oligosaccharide chain, as mono or bidesmosidic structures. Many of these compounds have been isolated and characterized during the last decades $[15,16]$. Herein we report the selection of isolated natural triterpene glycosides as structures bearing different glycosyl moieties and their immunochemical characterization in MS patients' sera. They have been used as antigenic probes, for the first time, to detect specific IgG and IgM antibodies in the sera of MS patients and normal blood donors (NBD) by using an enzyme-linked immunosorbent assay (ELISA). Results were compared with the CSF114(Glc)-based ELISA [17].

Five natural triterpene glycosides, selected for the complexity of their osidic part (compounds 1-5, \& Fig. 1), were used for the first time to detect specific IgG and IgM antibodies in MS patients' sera and NBD by ELISA: 3-O-( $\beta$-D-glucopyranosyl)presenegenin $28-\{0-\beta$-D-galactopyranosyl- $(1 \rightarrow 4)-O$-[ $\beta$-D-glucopyranosyl-( $1 \rightarrow 3)]-0-\beta$-D-xylopyranosyl-( $\rightarrow 4)-0$ - $\alpha$-L-rhamnopyranosyl-( $1 \rightarrow 2)-\{4-0$-[(E)-3,4-dimethoxycinnamoyl]\}- $\beta$-D-fucopyranosyl ester (1) isolated from Polygala arenaria Willd. (Polygalaceae) [18], 3-O-( $\beta$-D-2-O-sulphonylquinovopyranosyl) quinovic acid-28-O-( $\beta$-D-glucopyranosyl) ester (zygophyloside E) (2) and 3-O-( $\beta$-D-2-O-sulphonylglucopyranosyl)quinovic acid-28-O-( $\beta$-D-glucopyranosyl) ester (zygophyloside G) (3) from Zygophyllum geslini Coss. (Zygophyllaceae) [19], 3-0- $\beta$-D-glucopyranosylpresenegenin (tenuifoline) (4) from Polygala ruwenzor- 


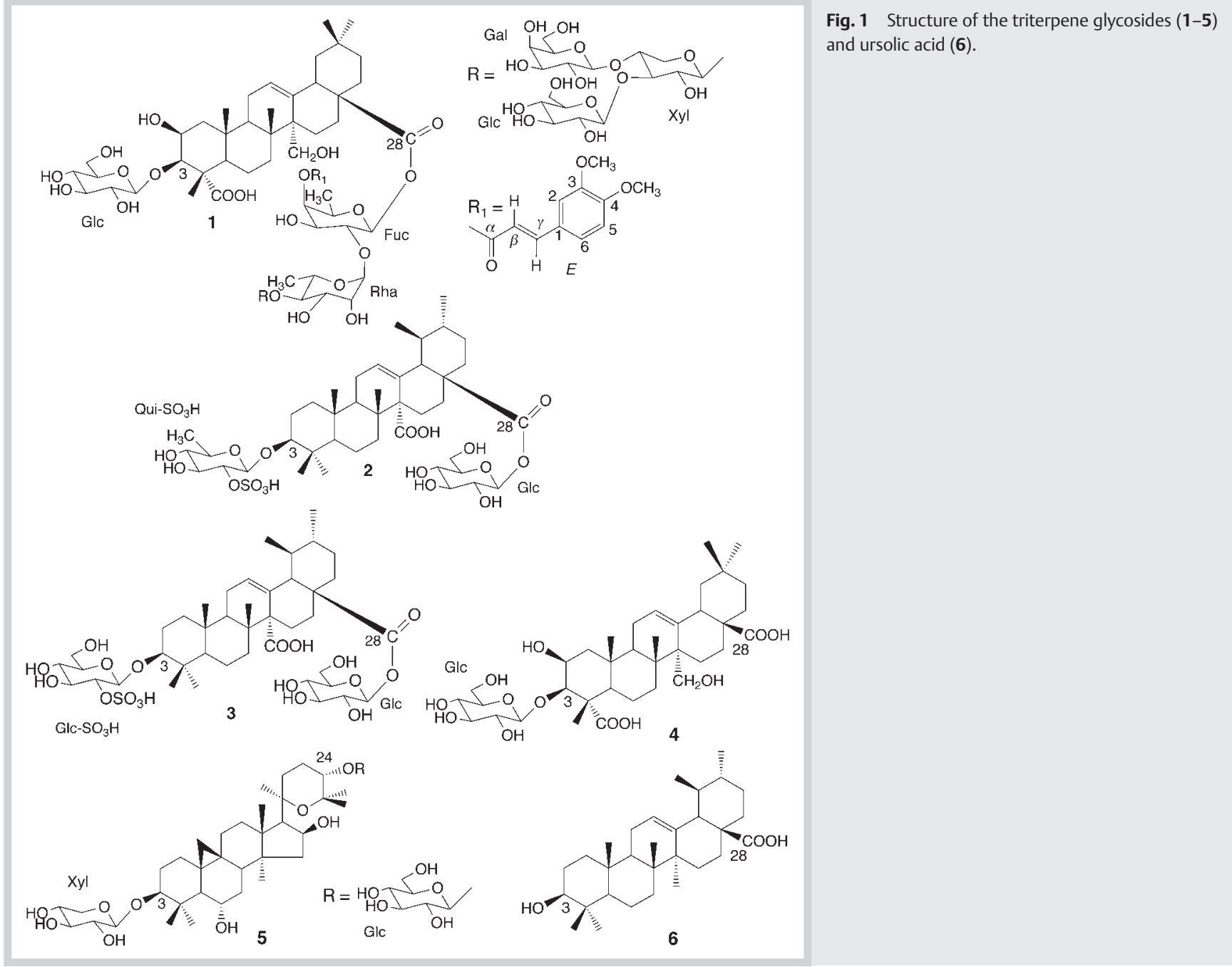

iensis Chod. [20], and 3-O- $\beta$-D-xylopyranosyl-24-O- $\beta$-D-glucopyranosyl-20,25-epoxycycloartane-3 $\beta, 6 \alpha, 16 \beta, 24 \alpha$-tetrol from Astragalus caprinus Maire (Fabaceae) [21,22]. In parallel, we used the glycopeptide CSF114(Glc)-based ELISA to compare antibody titers. The unglucosylated peptide CSF114 was employed as a negative control. Moreover, we tested the ursolic acid (6) containing a similar structure of compounds $\mathbf{1 - 5}$ but lacking glycosyl moieties in order to define if antibody responses are directed to glycosylated sites. The isolated compounds were coated in ELISA microplates to evaluate antibody titers in the sera. For each antigen, IgG and IgM measurements and statistical analysis were performed separately. Data distribution of IgG antibody responses to glycopeptide CSF114(Glc), its unglucosylated counterpart CSF114, and compounds 1-6 can be observed in $\bullet$ Fig. 2 . The glycopeptide CSF114(Glc) displayed the better performance, but the two-tailed Mann-Whitney test revealed no significant differences between patients and healthy control populations for CSF114(Glc), CSF114, triterpene glycosides $\mathbf{1 - 5}$, and ursolic acid 6 ( $\mathrm{p}$ values $>0.05$ ).

Column scatter of data reported in $\odot$ Fig. 3 summarizes IgM serological results obtained in ELISA for all of the compounds analyzed. All compounds presented lower mean values for NBD than for MS populations, except ursolic acid (6). This result highlighted the important role of the glycosylation for specific MS antibody recognition. Moreover, the glycopeptide CSF114(Glc) presented the best performance with significant differences between patients and controls (two-tailed Mann-Whitney test, p value $<0.001$ ), evidencing the role of the amide bond linkage.

The receiver operating characteristic (ROC) analysis was employed to compare the discrimination power of the compounds, and the different cutoff values, sensitivity, specificity, and likelihood ratios were also calculated [23]. Selected cutoff values, sensitivities, and the rest of the statistical parameters are summarized in Table 1. The characteristics of the curves, their shape and steepness, and their underlying area provide evidence that the selected natural triterpene glycosides are able to identify MS patients with a high degree of confidence at the selected cutoff values. These values are in any case lower than those obtained with the glycopeptide CSF114(Glc) as a synthetic antigenic probe highlighting the role of the N-glucosyl linkage in the antibody recognition. The diagnostic power of ursolic acid $\mathbf{6}$ was, in any case, lower than those presented by the triterpene glycosides 1 5.

A statistical analysis based on the Spearman ranking coefficient correlation was performed to determine cross-reactivity of the antibody titers for the glucopeptide CSF114(Glc), the triterpene glycosides 1-5, and the ursolic acid $\mathbf{6}$. The calculated Spearman $r$ values for compounds $\mathbf{1 - 4}$ to the glucopeptide CSF114(Glc) range from $0.499-0.742$, demonstrating a good correlation of antibody reactivities (calculated $\mathrm{p}$ values $<0.05$, two-tailed Spearman 


\section{$\lg \mathbf{G}$}
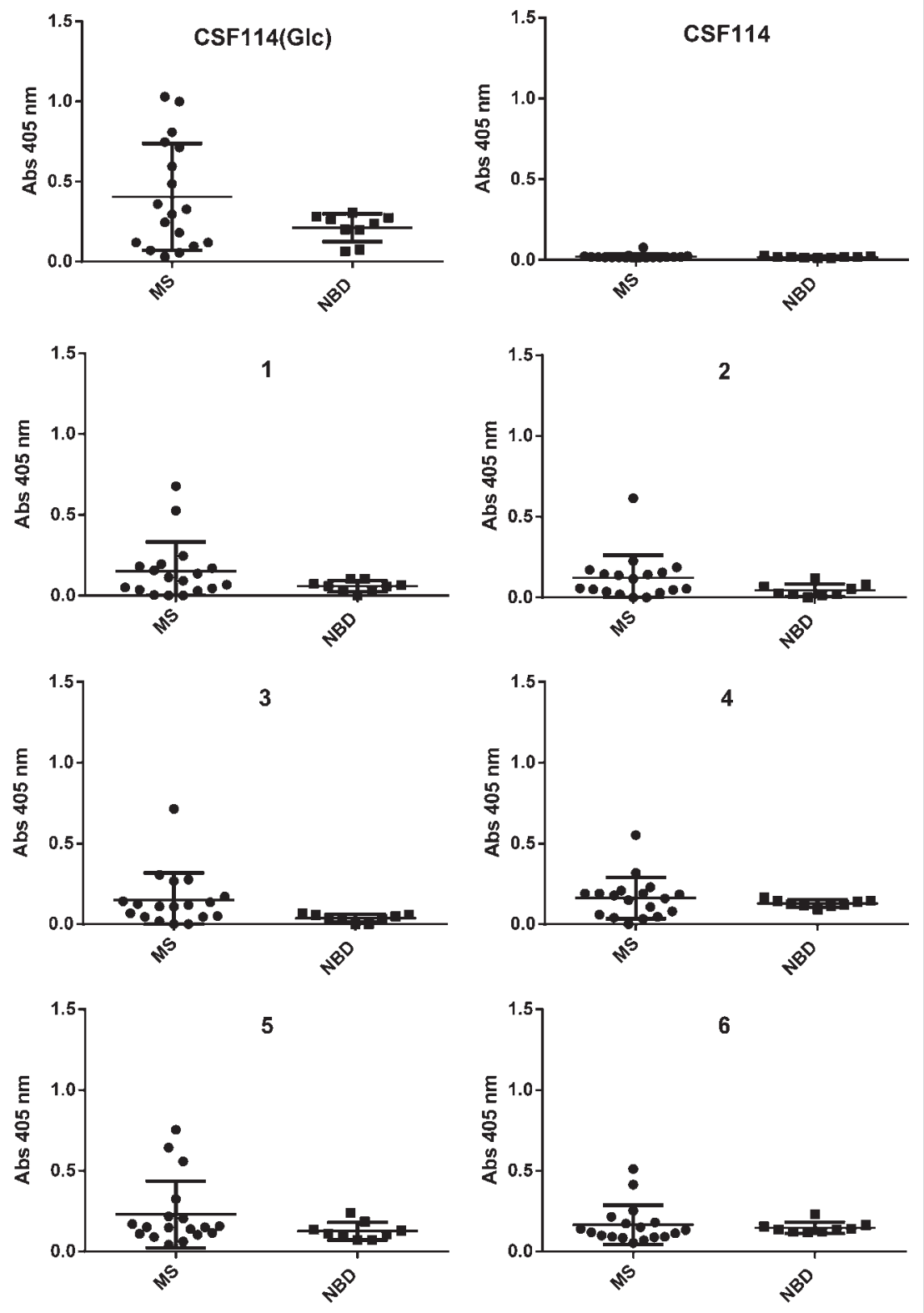

Fig. 2 Data distribution of IgG antibody responses to $\operatorname{CSF} 114(\mathrm{Glc})$, the unglucosylated peptide CSF114, saponins 1-5, and the ursolic acid $\mathbf{6}$ in the sera of multiple sclerosis patients (MS) and normal blood donors (NBD) determined by ELISA. Data are reported as absorbance at $405 \mathrm{~nm}$ of sera diluted $1: 100$. The lines represents the means values with the SD. Mann-Witney evaluation showed that the data do not differ significantly between the MS patient population and NBD for all compounds tested ( $p$ value $>0.05$ ) test). On the other hand, a lower correlation was observed with compound 5 ( $r=0.256$, p value $>0.05$, two-tailed Spearman test) and no correlation was observed with the ursolic acid 6 $(\mathrm{r}=-0.032$, $\mathrm{p}$ value $>0.05$, two-tailed Spearman test $)$.

Therefore, we investigated the correlation between the five natural triterpene-type glycoside structures and their biological activity highlighting their similarities with the CSF114(Glc) structure. From a structural point of view, compounds $\mathbf{1 - 3}$ and 5 are bidesmosides, with an O-heterosidic linkage with only one sugar at the $\mathrm{C}-3$ position of the aglycon. In particular, they are glucopyranosyl, sulfated glucopyranosyl, sulfated quinovopyranosyl, and xylopyranosyl moieties. Even if the number of the tested molecules till now is not statistically relevant, we can hypothesize the relevant role of the ester carboxyl function at the C-28 position in compounds 1-3 in IgM recognition. Among the bidesmosidic compounds with a C-28 ester (1-3), the best performance was obtained with 2 followed by $\mathbf{3}$, emphasizing the role of the size of the sugar, which is reduced at only one glucopyranosyl moiety in the natural triterpene glycosides $\mathbf{2}$ and 3. Moreover, analyzing the nature of the sugar at the $\mathrm{C}-3$ position of the aglycon, we expected the best results with the glucosylated triterpenes. In fact, the ursolic acid $\mathbf{6}$, presenting the scaffold of compounds 1-5 but lacking the glycosyl moieties, was not able to identify specific MS antibodies. This result demonstrated that ELISA based on trierpene glycoside structures bearing glycosyl moieties does not disrupt antibody recognition. On the basis of these results, the best structures to be selected should be: monodesmosidic with only one glucopyranosyl moiety in position C-3 or bidesmosidic with one sugar, sulfated or not, at the $\mathrm{C}-3$ position and a substitution with only one glucopyranosyl moiety at the C-24 or C-28 po- 


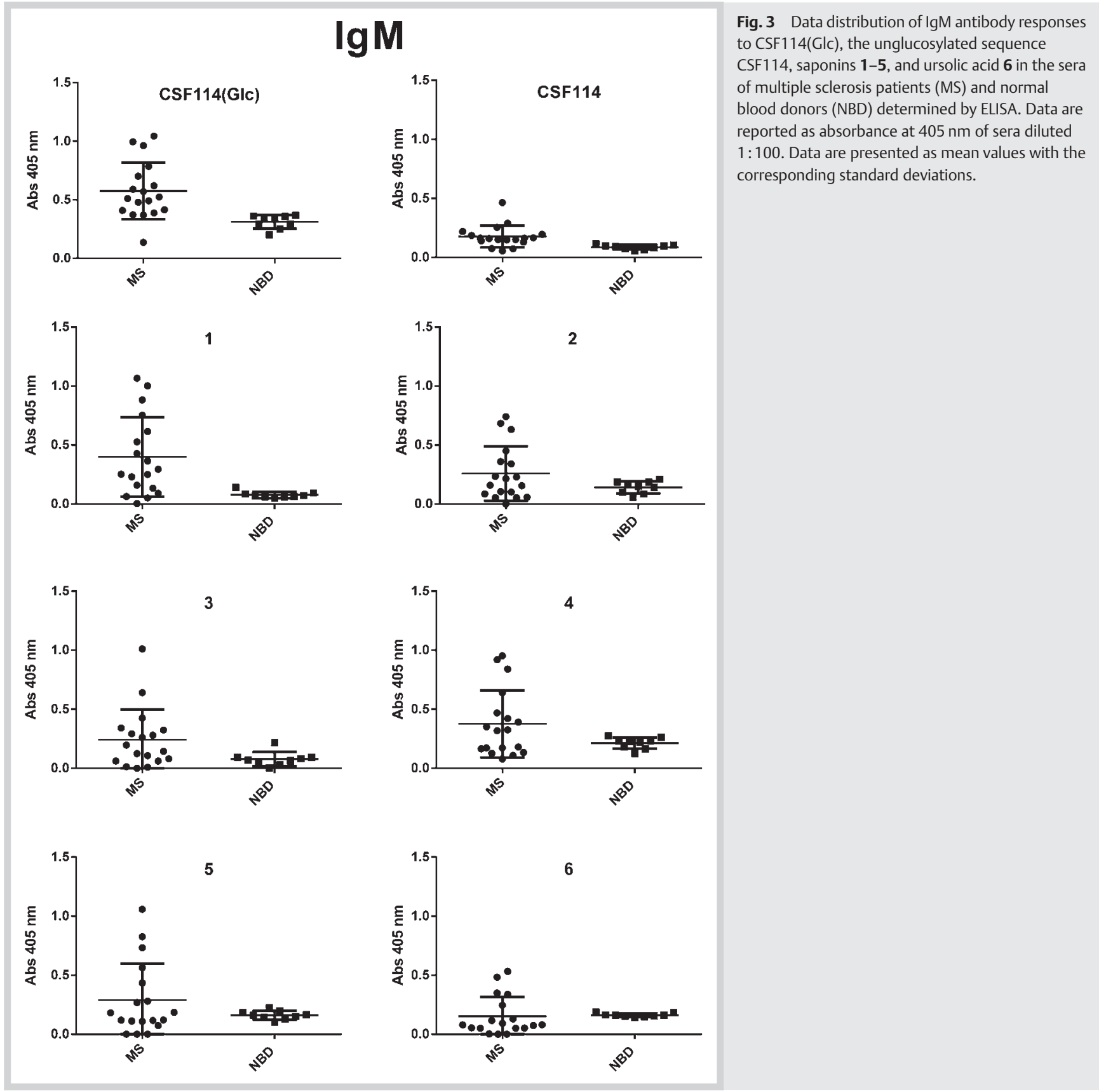

sition. From a chemotaxonomic point of view, desertic plants as the Zygophyllaceae family represent a potential source of sulfated triterpene glycosides [19,24].

We report for the first time the use of triterpene glycosides as natural antigenic probes. These could allow a screening by immunoenzymatic assays to evaluate serum titers recognition by complex sugar epitopes. In fact, the data obtained confirmed the importance of carbohydrate structures in antibody recognition.

\section{Material and Methods}

$\nabla$

\section{Plant material}

The harvest of $P$. arenaria and $P$. ruwenzoriensis from the Democratic Republic of Congo, Z. geslini from the Algerian Sahara, and A. caprinus from Monastir, Tunisia, is detailed in the references [18-22].

\section{Extraction, isolation, and characterization}

The extraction, isolation, and the physicochemical and spectroscopic data of the triterpene glycosides (1-5, $\bullet$ Fig. $\mathbf{1})$ have been previously reported in references [18-22] (see Supporting Information). Ursolic acid (6) was purchased from Sigma-Aldrich.

\section{Glycopeptide synthesis}

The glycopeptide antigen CSF114(Glc) and its unglucosylated analog were prepared by microwave-assisted solid-phase peptide synthesis. The glycopeptide was purified to homogeneity by solid-phase extraction and reverse-phase high-pressure liquid chromatography (HPLC), and further characterized by mass spectrometry and analytical HPLC, as described elsewhere [25]. 
Table 1 Values obtained from ROC analysis of compounds 1-6 and glycopeptide CSF114(Glc) for the area under the curve, p value, established cutoff, and the corresponding sensitivity, specificity, and likelihood ratio.

\begin{tabular}{|c|c|c|c|c|c|c|c|c|}
\hline \multirow[t]{2}{*}{ Compound } & \multicolumn{4}{|c|}{ Area under curve (AUC) } & \multicolumn{4}{|c|}{ Criterion value and coordinates } \\
\hline & AUC & $\begin{array}{l}\text { Standard } \\
\text { error (SE) }\end{array}$ & $\begin{array}{l}\text { 95\% Confidence } \\
\text { interval }\end{array}$ & $P$ value & Cutoff & Sensitivity (\%) & Specificity (\%) & $\begin{array}{l}\text { Likelihood } \\
\text { ratio }\end{array}$ \\
\hline 1 & 0.839 & 0.08 & $0.68-0.99$ & 0.0047 & $>0.115$ & $\begin{array}{l}77.8 \% \\
52.4-93.6\end{array}$ & $\begin{array}{l}88.9 \% \\
51.8-99.7\end{array}$ & 7.00 \\
\hline 2 & 0.624 & 0.11 & $0.41-0.83$ & 0.3037 & $>0.199$ & $\begin{array}{l}50.0 \% \\
26.0-73.9\end{array}$ & $\begin{array}{l}88.9 \% \\
51.8-99.7\end{array}$ & 4.50 \\
\hline 3 & 0.726 & 0.10 & $0.54-0.92$ & 0.0605 & $>0.101$ & $\begin{array}{l}66.7 \% \\
41.0-86.6\end{array}$ & $\begin{array}{l}88.9 \% \\
51.8-99.7\end{array}$ & 6.00 \\
\hline 4 & 0.617 & 0.11 & $0.40-0.83$ & 0.3285 & $>0.269$ & $\begin{array}{l}55.6 \% \\
30.8-78.5\end{array}$ & $\begin{array}{l}88.9 \% \\
51.7-99.7\end{array}$ & 5.00 \\
\hline 5 & 0.500 & 0.11 & $0.28-0.72$ & 1.0 & $>0.212$ & $\begin{array}{l}38.9 \% \\
17.3-64.3\end{array}$ & $\begin{array}{l}88.9 \% \\
51.8-99.7\end{array}$ & 3.50 \\
\hline 6 & 0.722 & 0.11 & $0.52-0.93$ & 0.0641 & $>0.186$ & $\begin{array}{l}11 \% \\
0.3-48.3\end{array}$ & $\begin{array}{l}72.2 \% \\
46.5-90.3\end{array}$ & 0.40 \\
\hline CSF114(Glc) & 0.94 & 0.05 & $0.84-1.05$ & 0.00022 & $>0.364$ & $\begin{array}{l}94.4 \% \\
72.7-99.9\end{array}$ & $\begin{array}{l}88.9 \% \\
51.8-99.7\end{array}$ & 8.50 \\
\hline
\end{tabular}

\section{Immunoenzymatic assays}

Twenty-seven sera were obtained for diagnostic purposes from MS patients (18) and healthy blood donors (9) who had given their informed consent, and stored at $-20^{\circ} \mathrm{C}$ until use. Patients were previously diagnosed after a lumbar puncture, MRI examination, and cerebrospinal analysis. Samples were preselected depending on their reactivity to CSF114(Glc). Antibody responses were determined in solid-phase ELISA (SP-ELISA).

The glycopeptide CSF114(Glc) and its unglycosylated analog were coated independently on 96-well activated polystyrene ELISA plates (NUNC Maxisorb SIGMA) using a solution of $10 \mu \mathrm{g} / \mathrm{mL}$ in pure carbonate buffer $0.05 \mathrm{M}$ at pH $9.6(100 \mu \mathrm{L} /$ well $)$ and incubated at $+4{ }^{\circ} \mathrm{C}$ overnight. After three washes with $150 \mathrm{mM} \mathrm{NaCl}$ solution containing $0.05 \%$ Tween 20 (washing buffer), nonspecific binding sites were blocked with fetal bovine serum (FBS) $10 \%$ in washing buffer ( $100 \mu \mathrm{L}$ per well) at room temperature for $60 \mathrm{~min}$. Sera diluted from $1: 100,1: 1000$, and $1: 10000$ in $10 \%$ FBS in washing buffer were incubated at $+4{ }^{\circ} \mathrm{C}$ overnight. After three washes, $100 \mu$ l of alkaline phosphatase conjugated antihuman IgM (diluted $1: 200$ in $150 \mathrm{mM} \mathrm{NaCl} / 0.05 \%$ Tween 20/10\% FBS) or IgG (diluted $1: 8000$ in $150 \mathrm{mM} \mathrm{NaCl} / 0.05 \%$ Tween $20 / 10 \%$ FBS; Sigma-Aldrich) were added to each well. After a 3-h incubation at room temperature and three washes, $100 \mu \mathrm{L} /$ well of $1 \mathrm{mg}$ $\times \mathrm{mL}^{-1}$ p-nitrophenyl phosphate (Sigma-Aldrich) in $10 \%$ diethanolamine substrate solution ( $\mathrm{pH} 9.8$ ) were added. After $30 \mathrm{~min}$, the reaction was stopped with $1 \mathrm{M} \mathrm{NaOH}(50 \mu \mathrm{L} /$ well $)$, and the absorbance was read in a multichannel ELISA reader (Tecan Sunrise) at $405 \mathrm{~nm}$. ELISA plates, coating conditions, reagent dilutions, buffers, and incubation times were preliminary tested [10]. Antibody levels are expressed as absorbance in arbitrary units at $405 \mathrm{~nm}$ (sample dilution $1: 100$ ).

The compounds 1-6 were coated on 96-well microplates BD Falcon $^{\mathrm{TM}}$ separately using a solution of $10 \mu \mathrm{g} / \mathrm{mL}$ in pure ethanol $(100 \mu \mathrm{L} /$ well $)$. The plates were then incubated at $+4^{\circ} \mathrm{C}$ overnight. Microplates coated with the triterpene glycosides were washed once with $150 \mathrm{mM} \mathrm{NaCl}$ without surfactants. The nonspecific binding sites were blocked with $10 \%$ FBS in $150 \mathrm{mM} \mathrm{NaCl}$ solution at room temperature for $60 \mathrm{~min}$. Then, the same protocol as above for the glycopeptide CSF114(Glc) was performed without using Tween 20.
Statistical analysis was performed using GraphPad software v4.0. Mann-Whitney tests were used to evaluate the predictive antibody values, and two-tailed p values were calculated. ROC curves were calculated for significant triterpene-type saponins and cutoff values were established.

\section{Supporting information}

The extraction and isolation of the test compounds are available as Supporting Information.

\section{Acknowledgement}

$\nabla$

This work was financially supported by the "Chaire d'Excellence" of Agence Nationale de la Recherche to A.M.P. (Project Pepkit: Development of peptide-based-diagnostic kits for autoimmune diseases, ANR-09-CEXC-013-01), in part, by the Fondazione Ente Cassa di Risparmio di Firenze (Italy), and by Regione Toscana (POR CReO FESR 2007-2013 ASSE 1- Linea di intervento 1.1.C Bando Regionale 2009).

\section{Conflict of Interest}

$\nabla$

All authors declare that they have no conflicts of interest.

\section{References}

1 Wymann MP, Schneiter R. Lipid signalling in disease. Nat Rev Mol Cell Biol 2008; 9: 162-176

2 Kim SH, Turnbull J, Guimond SJ. Extracellular matrix and cell signalling: the dynamic cooperation of integrin, proteoglycan and growth factor receptor. J Endocrinol 2011; 209: 139-151

3 Nobile-Orazio E, Giannotta C. Testing for anti-glycolipid IgM antibodies in chronic immune-mediated demyelinating neuropathies. J Peripher Nerv Syst 2011; 16: 18-23

4 Miyahara K, Nouso K, Saito S, Hiraoka S, Harada K, Takahashi S, Morimoto Y, Kobayashi S, Ikeda F, Miyake Y, Shiraha H, Takaki A, Okada H, Amano $M$, Hirose K, Nishimura S, Yamamoto K. Serum glycan markers for evaluation of disease activity and prediction of clinical course in patients with ulcerative colitis. PLoS One 2013; 8: e74861

5 Kaul A, Hutfless S, Liu L, Bayless TM, Marohn MR, Li X. Serum anti-glycan antibody biomarkers for inflammatory bowel disease diagnosis and progression: a systematic review and meta-analysis. Inflamm Bowel Dis 2012; 18: 1872-1884 
6 Alexander JS, Zivadinov R, Maghzi AH, Ganta VC, Harris MK, Minagar A. Multiple sclerosis and cerebral endothelial dysfunction: Mechanisms. Pathophysiology 2011; 18: 3-12

7 Doyle HA, Mamula MJ. Post-translational protein modification in antigen recognition and autoimmunity. Trends Immunol 2001; 22: 443449

8 Lolli F, Mulinacci B, Carotenuto A, Bonetti B, Sabatino G, Mazzanti B, D'Ursi AM, Novellino E, Pazzagli M, Lovato L, Alcaro MC, Peroni E, PozoCarrero MC, Nuti F, Battistini L, Borsellino G, Chelli M, Rovero P, Papini $A M$. An N-glucosylated peptide detecting disease-specific autoantibodies, biomarkers of multiple sclerosis. Proc Natl Acad Sci U S A 2005; 102: 10273-10278

9 Marta CB, Oliver AR, Sweet RA, Pfeiffer SE, Ruddle NH. Pathogenic myelin oligodendrocyte glycoprotein antibodies recognize glycosylated epitopes and perturb oligodendrocyte physiology. Proc Natl Acad Sci U S A 2005; 102: 13992-13997

10 Lolli F, Mazzanti B, Pazzagli M, Peroni E, Alcaro MC, Sabatino G, Lanzillo $R$, Brescia Morra V, Santoro L, Gasperini C, Galgani S, D'Elios MM, Zipoli $V$, Sotgiu S, Pugliatti M, Rovero P, Chelli M, Papini AM. The glycopeptide CSF114(Glc) detects serum antibodies in multiple sclerosis. J Neuroimmunol 2005; 167: 131-137

11 Real-Fernández F, Colson A, Bayardon J, Nuti F, Peroni E, Meunier-Prest R, Lolli F, Chelli M, Darcel C, Jugé S, Papini AM. Ferrocenyl glycopeptides as electrochemical probes to detect autoantibodies in multiple sclerosis patients' sera. Biopolymers 2008; 90: 488-495

12 Carotenuto A, D'Ursi AM, Mulinacci B, Paolini I, Lolli F, Papini AM, Novellino E, Rovero P. Conformation-activity relationship of designed glycopeptides as synthetic probes for the detection of autoantibodies, biomarkers of multiple sclerosis. J Med Chem 2006; 49: 5072-5079

13 Carotenuto A, Alcaro MC, Saviello MR, Peroni E, Nuti F, Papini AM, Novellino $E$, Rovero $P$. Designed glycopeptides with different $\beta$-turn types as synthetic probes for the detection of autoantibodies as biomarkers of multiple sclerosis. J Med Chem 2008; 51: 5304-5309

14 Nuti F, Peroni E, Real-Fernández F, Bonache MA, Le Chavalier-Isaad A, Chelli M, Lubin-Germain N, Uziel J, Rovero P, Lolli F, Papini AM. Posttranslationally modified peptides efficiently mimicking neoantigens: a challenge for theragnostics of autoimmune diseases. Biopolymers 2010; 94: 791-799

15 Lacaille-Dubois MA, Mitaine-Offer AC. Triterpene saponins from Polygalaceae. Phytochem Rev 2005; 4: 139-149

16 Osbourn A, Gossb RJ, Field RA. The saponins - polar isoprenoids with important and diverse biological activities. Nat Prod Rep 2011; 28 : $1261-1268$

17 Papini AM. Simple test for multiple sclerosis. Nat Med 2005; 11: 13

18 Mitaine-Offer AC, Miyamoto T, Laurens V, Delaude C, Lacaille-Dubois MA. New acylated triterpene saponins from Polygala arenaria. Helv Chim Acta 2003; 86: 2404-2413

19 Smati D, Mitaine-Offer AC, Miyamoto T, Hammiche V, Lacaille-Dubois MA. Ursane-type triterpene saponins from Zygophyllum geslini. Helv Chim Acta 2007; 90: 712-719

20 Mitaine-Offer AC, Miyamoto T, Jolly C, Delaude C, Lacaille-Dubois MA. Triterpene saponins from four species of the Polygalaceae family. Helv Chim Acta 2005; 88: 2986-2995
21 Semmar N, Fenet B, Lacaille-Dubois MA, Gluchoff-Fiasson K, Chemli R, Jay M. Two new glycosides from Astragalus caprinus. J Nat Prod 2001; 64: 656-658

22 Mitaine-Offer AC, Miyamoto T, Semmar N, Jay M, Lacaille-Dubois MA. A new oleanane glycoside from the roots of Astragalus caprinus. Magn Reson Chem 2006; 44: 713-716

23 Habbema JD, Eijkemans R, Krijnen J, Knottnerus JE. Analysis of data on the accuracy of diagnostic tests. In: Knottnerus JE, editor. The evidence base of clinical diagnosis. London: BMJ Books; 2002: 117-143

24 Pöllmann K, Gagel S, Elgamal MH, Shaker KH, Seifert K. Triterpenoid saponins from the roots of Zygophyllum species. Phytochemistry 1997; 44: 485-489

25 Rizzolo F, Sabatino G, Chelli M, Rovero P, Papini AM. A convenient microwave-enhanced solid-phase synthesis of difficult peptide sequences: Case study of gramicidin A and CSF114(Glc). Int J Pept Res Ther 2007; 13: $203-208$

received July 11,2015

revised November 20, 2015

accepted November 30, 2015

Bibliography

DOI http://dx.doi.org/10.1055/s-0035-1568263

Published online February 5, 2016

Planta Med Lett 2016; 3: e2-e7

(c) Georg Thieme Verlag KG Stuttgart · New York .

ISSN 2199-157X

Correspondence

Prof. Marie-Aleth Lacaille-Dubois

Laboratoire de Pharmacognosie

EA 4267 FDE

UFR Sciences de Santé

Université de Bourgogne Franche-Comté

7 Bd Jeanne d'Arc, BP 87900

21079 Dijon cedex

France

Phone: +33380393229

Fax: +33380393300

m-a.lacaille-dubois@u-bourgogne.fr

Prof. Anna-Maria Papini

Department of Chemistry "Ugo Schiff" and NEUROFARBA Department, Section of Pharmaceutical Sciences and Nutraceutics

University of Florence

Via della Lastruccia 13

50019 Sesto Fiorentino

Italy

Phone: +390554573561

Fax: +390554574912

annamaria.papini@unifi.it

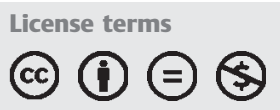

